\title{
Dual Function of Silver Nanoparticles as Matrix Extracell Removal and Antimicrobial Agent in Polymycrobial Biofilms
}

\author{
Mei Shirli Yasinta, Hera Lisna Ginawati, Nira Ambar Arum, Harini Nur Hikmah, Sri Sumarsih, Mochamad \\ Zakki Fahmi, and Afaf Baktir
}

Department of Chemistry, Airlangga University, Kampus C Mulyorejo, Surabaya 60115, Indonesia

\author{
*Corresponding author: \\ email:afafi2001@yahoo.com
}

Received: December 9, 2019

Accepted: August 5, 2020

DOI: $10.22146 /$ ijc. 52355

\begin{abstract}
Candida albicans often form polymicrobial biofilms along with pathogenic microbes. Silver nanoparticles (AgNPs) were well known to have strong antimicrobial activity. However, their effect on polymicrobial biofilms and the mechanism has never been reported. This study aimed to synthesize AgNPs and study their effects on polymicrobial biofilm represented by C. albicans-E. coli biofilm. Polymicrobial biofilms, formed by clinical isolates of $\mathrm{C}$. albicans and $\mathrm{E}$. coli, were developed from the standardized suspensions of each strain by culturing flat-bottom 96-well microtiter plates for 48 then treated with AgNPs. Cell viability was assessed using the tetrazolium salt reduction assay; the extent of biofilm formation was measured by crystal violet staining. AgNPs reduced the polymicrobial biofilm in two ways: by degrading the extracellular matrix and killing both C. albicans and E. coli. The results showed AgNPs are a potential new approach for developing potent anti-biofilms.
\end{abstract}

Keywords: silver nanoparticle; polymicrobial biofilm; C. albicans; E. coli; anti-biofilm

\section{- INTRODUCTION}

Research on biofilms has changed the paradigm of the life of microorganisms. Microorganisms can develop biofilms, which are extracellular matrix-coated communities of microbial cells attached to the host surface [1]. Biofilms are the predominant growth state of many microorganisms [2-4]. According to the National Institute of Health, pathogenic biofilms in the United States played a role in more than $80 \%$ of microbial infection cases [4]. Some infection cases related to biofilms included cystic fibrosis, catheter infection, endocarditis, chronic prostatitis, periodontal disease, and ear infections [5,6]. Based on the in vitro studies, microorganisms in the form of biofilms can avoid host defense systems, and they are 10-10 times more resistant to antimicrobial agents than their free (planktonic) cells $[1,7]$. In nature, most microorganisms do not live as single species [8]. They tend to live as a community with other species. Thus, naturally occurring biofilms contain more than one microbial species, called polymicrobial biofilms [8].
Candida albicans (C. albicans) is one type of microbe that is capable of building a biofilm and causing infection in humans [9]. It is the most prevalent fungal species in the micro-ecosystem of human microbiota [10]. It is estimated that the polymicrobial biofilms of $C$. albicans cause $27-56 \%$ of blood-borne nosocomial infections. Candida is often found in the form of polymicrobial biofilms and various types of pathogenic microbes, including Staphylococcus, Pseudomonas, and Escherichia coli. For example, Candida interacts with Streptococcus, Lactobacilli, Porphyromonas gingivalis in oral sites. Candida and Cryptococcus interact with various gram-negative and -positive bacteria in the lower reproductive tract. Candida interacts with grampositive and -negative bacteria (usually Staphylococcus) and dermatophyte species on skin sites and vascular catheters. Candida also interacts with Enterobacteriaceae and Enterococcus in intra-abdominal sites, as well as with Enterobacteriaceae, Escherichia coli (E. coli), and Enterobacter faecalis in the urinary tract [11]. 
Polymicrobial biofilms have been shown to have several advantages for the multiple species residing within them. These include increased tolerance to antimicrobial agents and increased virulence [12], increasing the difficulty of inhibiting these biofilms. This poses a major clinical issue for human health $[2,13]$. Previous studies have shown that E. coli and C. albicans coexist in polymicrobial biofilms isolated from endotracheal tubes and urine catheters [14-15]. This polymicrobial biofilm is thought to increase resistance to the antibiotic ofloxacin [16]. Our current study focuses on identifying a method to inhibit C. albicans-E. coli biofilms.

For this purpose, we used silver nanoparticles (AgNPs) as they have been reported to have both antibacterial and antifungal activities [16-17]. Moreover, other studies suggest that AgNPs can inhibit the in vitro formation of biofilms by various microbial species, such as $P$. aeruginosa, $S$. aureus, $S$. epidermis, $S$. pneumonia, and $S$. flexneri $[5,18]$. However, the mechanism underlying the activity of AgNPs against polymicrobial biofilms is not understood in detail. Thus, the present study aimed to synthesize AgNPs and study their effects on polymicrobial biofilm represented by C. albicans-E. coli biofilm.

AgNPs were known less reactive than $\mathrm{Ag}^{+}$, making their antibacterial activity lower than $\mathrm{AgNO}_{3}$. The inhibitory effect of $\mathrm{AgNO}_{3}$ on Pseudomonas putida mt-2 growth was 1,600 times more than AgNP [19]. On the other hand, extracellular matrix-covered bacterial cells in the biofilm structure more easily penetrated by nanoparticles or nano-sized molecules than that of $\mathrm{Ag}^{+}$, which tend to form cluster structures causing low penetration ability. Based on this fact, we will compare the toxicity of $\mathrm{AgNPs}$ and $\mathrm{AgNO}_{3}$ against bacteria and fungi in polymicrobial biofilm protected by wrapped extracellular matrix. Indeed, it is important to compare the effectiveness of AgNPs and $\mathrm{AgNO}_{3}$ for eradicating $C$. albicans-E. coli biofilm.

\section{- EXPERIMENTAL SECTION}

\section{Materials}

Silver nitrate $\left(\mathrm{AgNO}_{3}\right)$ and tri-sodium citrate $\left(\mathrm{C}_{6} \mathrm{H}_{5} \mathrm{O}_{7} \mathrm{Na}_{3}\right)$ were purchased from Sigma Aldrich. The $C$. albicans (ATCC 10231) and E. coli (ATCC 25992) were purchased from the Surabaya Health Laboratory Center (Balai Besar Laboratorium Kesehatan Surabaya). The cultures of C. albicans were grown overnight in yeast extract-peptone-dextrose $[1 \%$ yeast extract $(\mathrm{BD}$ Biosciences), $2 \%$ peptone (Oxoid Ltd.), and 2\% dextrose (Conda Pronadisa)] at $37^{\circ} \mathrm{C}$. The cultures of E. coli were grown overnight in Luria Bertani $[1 \%$ tryptone (Himedia); $0.5 \%$ yeast extract (BD bioscience); and $1 \%$ $\mathrm{NaCl}$ (Merck)] medium at $37^{\circ} \mathrm{C}$. SDB medium was used to create a polymicrobial biofilm in a 96 -well microtiter plate. SYTO-59 dye, FITC, and Con A to visualize E. coli, C. albicans extracellular matrix, respectively.

\section{Procedure}

\section{Synthesis of AgNPs}

$\mathrm{C}_{6} \mathrm{H}_{5} \mathrm{O}_{7} \mathrm{Na}_{3}$ and $\mathrm{AgNO}_{3}$ of analytical grades were used. AgNPs were prepared using a chemical reduction method. Briefly, $100 \mathrm{~mL}$ of $1-\mathrm{mM} \mathrm{AgNO}_{3}$ was heated to the boiling point. Then, $5 \mathrm{~mL}$ of $1 \% \mathrm{C}_{6} \mathrm{H}_{5} \mathrm{O}_{7} \mathrm{Na}_{3}$ was added dropwise to this solution. The solution was vigorously stirred during this process. Then the solution was then heated to form a pale-yellow solution. This solution was then stirred until it cooled to room temperature [19].

\section{Growth of C. albicans-E. coli biofilms}

The overnight cultures of $C$. albicans and E. coli with optical densities of 0.5 at $600 \mathrm{~nm}$ (OD600) were diluted in the SDB medium. Each cell suspension of $75 \mu \mathrm{L}$ was added to the wells of a round-bottom microtiter plate (TPP, Trasadingen, Switzerland). These C. albicans-E. coli biofilms in these wells were allowed to grow by adding SDB medium under non-shaking conditions at $37^{\circ} \mathrm{C}$ for $48 \mathrm{~h}$. After the incubation period, the culture was further diluted with a fresh SDB medium.

\section{Treatment of C. albicans-E. coli biofilms with AgNP and $\mathrm{AgNO}_{3}$}

C. albicans-E. coli biofilms were divided into three treatment groups. The first group was treated with AgNPs (50 and $100 \mu \mathrm{L}$ ), the second group was treated with $\mathrm{AgNO}_{3}$ (50 and $100 \mu \mathrm{L}$ ), and the third group was left the untreated group and used as a control. Microtiter plates were incubated for $6 \mathrm{~h}$ at $37^{\circ} \mathrm{C}$. After incubation, 
the wells were washed twice with $0.2 \mathrm{~mL}$ of PBS (pH 7.2) to remove non-adherent cells.

\section{Crystal violet assay}

C. albicans-E. coli biofilms that were adhered to the walls and bottoms of the wells were stained with $0.01 \%$ crystal violet for $30 \mathrm{~min}$. Subsequently, the excess stain was washed off twice with sterile water. The wells were then fixed with $30 \%$ acetate acid for $20 \mathrm{~min}$; the solution $(100 \mu \mathrm{L})$ was transferred to a new microtiter plate for obtaining absorbance readings. The optical densities (ODs) of the stained and adherent C. albicans-E. coli biofilms were collected using a micro ELISA auto reader at a wavelength of $595 \mathrm{~nm}$. The average OD of the sterile medium was calculated and subtracted from all the treatment groups' ODs. All steps were conducted at room temperature.

\section{Tetrazolium salt reduction (XTT) assay}

After all treatments, the XTT assay was performed as a measure of metabolic activity to estimate the burden of viable cells. Using a multichannel pipette, $40 \mu \mathrm{L}$ of XTT solution was added along with $2 \mu \mathrm{L}$ of menadione and 158 $\mu \mathrm{L}$ of PBS to each well containing a pre-washed $C$. albicans-E. coli biofilm. Plates were then covered in aluminum foil and incubated in the dark for $2 \mathrm{~h}$ at $37^{\circ} \mathrm{C}$. Subsequently, the plates were then uncovered, and a multichannel pipette was used to remove $80 \mu \mathrm{L}$ of the resulting colored supernatant from each well. This supernatant was transferred into the corresponding wells of a new microtiter plate. All plates were read in a microtiter plate reader at $490 \mathrm{~nm}$.

\section{Characterizations}

Using a UV-1800 spectrophotometer (Shimadzu, Japan), a specific ultraviolet (UV)-Vis spectrum was obtained for AgNPs at a wavelength of 300-600 nm. AgNPs size distribution was assessed using a Zeta Sizer (Malvern Zeta Sizer Nano ZS90). For the visual observation of C. albicans-E. coli biofilms, biofilm samples were analyzed using SEM and CLSM (Olympus type FV1000). For this, both control and treated $C$. albicans-E. coli biofilms were prepared according to the methods described above with modifications in spider medium for growing C. albicans-E. coli biofilms on cellulose nitrate filter membranes. The samples were dried overnight at $37^{\circ} \mathrm{C}$. Samples were then dehydrated through a series of graded ethanol (50\% for $10 \mathrm{~min}, 70 \%$ for $10 \mathrm{~min}$, and $96 \%$ for $20 \mathrm{~min}$ ). The dried C. albicansE. coli biofilms were finally coated with palladium, and their surface was observed using SEM. For CLSM, samples were washed with PBS, stained with SYTO stains for E. coli (red); concanavalin A (Con A) for C. albicans-E. coli biofilm matrix (green); tetramethylrhodamine for C. albicans (red); and fluorescein isothiocyanate (FITC) for C. albicans (green).

\section{- RESULTS AND DISCUSSION}

Naturally occurring biofilms contain more than one microbial species and are known as polymicrobial biofilms. Such biofilms are more harmful than monomicrobial biofilms. An antimicrobial agent must be able to degrade and penetrate the extracellular matrix to reach bacterial and fungal cells coexisting in these biofilms to incapacitate polymicrobial biofilms. To solve this problem, in our study, we synthesized AgNPs for use as antimicrobial agents.

AgNPs were successfully synthesized using a chemical reduction method. A change in the solution color characterized AgNPs formation from clear to pale yellow. Thus, the AgNPs formed were stable for 3 weeks, as confirmed by a weekly absorbance examination using a UV-Vis spectrophotometer. AgNPs characterized using a UV-Vis spectrophotometer showed a maximum wavelength at $431.5 \mathrm{~nm}$ (Fig. 1(c)), indicating the formation of AgNPs [19]. DLS conducted to determine AgNPs size revealed that the average size of synthesized AgNPs was $20 \mathrm{~nm}$.

The IR spectrum of AgNPs determined using FTIR in Fig. 1(b) revealed some peaks that were following that of AgNPs in a previous study [20]. A peak observed at $3431.36 \mathrm{~nm}^{-1}$ corresponded to the $\mathrm{OH}$ functional group from $\mathrm{H}_{2} \mathrm{O}$ (solvent). A peak at $1614.42 \mathrm{~nm}^{-1}$ corresponded to the $\mathrm{C}=\mathrm{O}$ functional group of $\mathrm{C}_{6} \mathrm{H}_{5} \mathrm{O}_{7} \mathrm{Na}_{3}$. Briefly, $\mathrm{C}_{6} \mathrm{H}_{5} \mathrm{O}_{7} \mathrm{Na}_{3}$ acts as a reducing agent and a stabilizing agent to prevent the agglomeration of AgNPs [21]. 
(a)

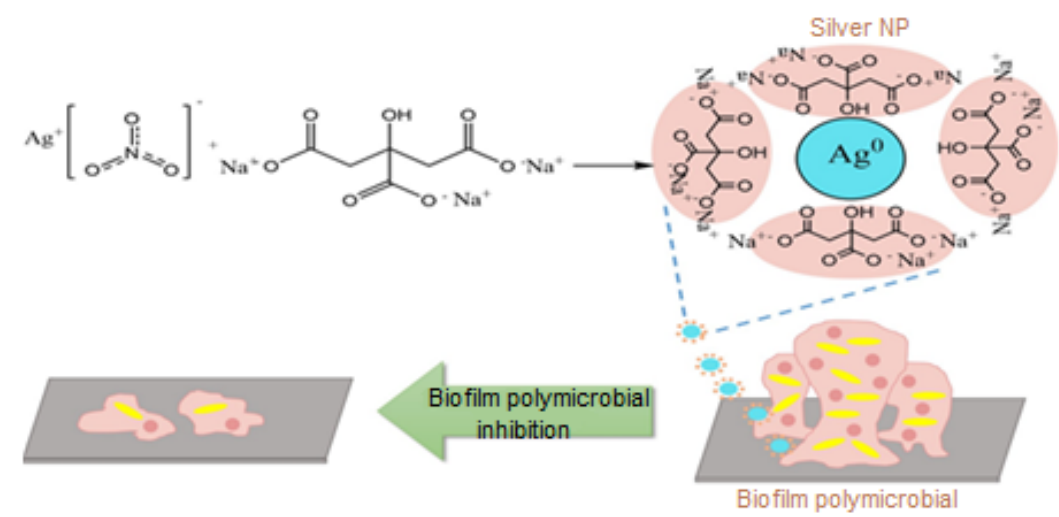

(b)

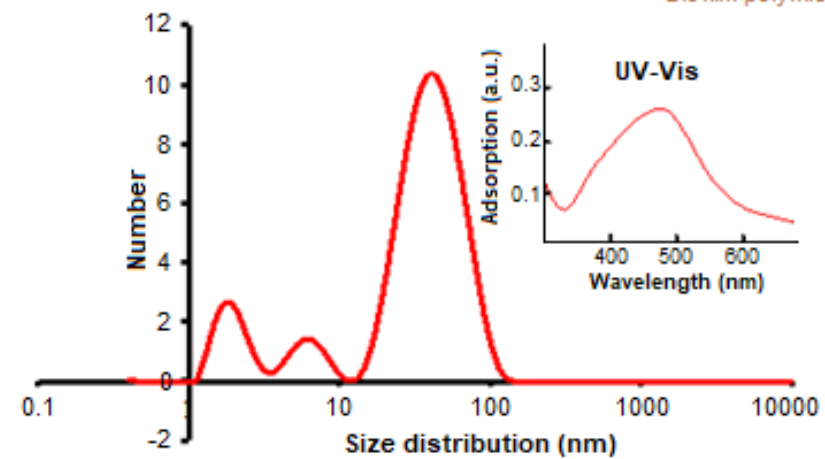

(c)

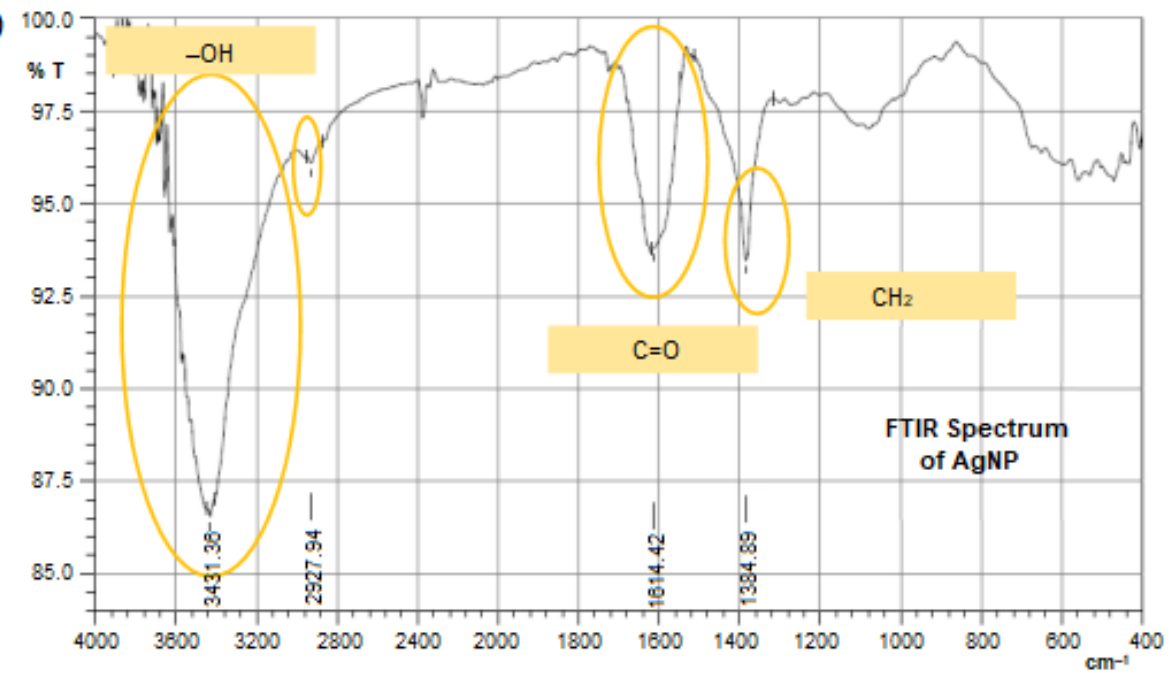

Fig 1. Synthesis, characterization, and application of AgNPs. (a) A schematic process on synthesis AgNPs and its application on biofilm polymicrobial inhibition. Characterization of AgNPs (b) FTIR of AgNPs (c) size distribution and UV-Vis spectra of AgNPs

AgNPs produced in this research were used for the treatment of polymicrobial biofilms. AgNPs can penetrate polymicrobial biofilms to hinder the growth of or eliminate residing microorganisms (Fig. 1). The ability of silver salts $\mathrm{AgNO}_{3}$ and AgNPs, to act as anti-biofilms against C. albicans-E. coli biofilms were also examined. Crystal violet assay was used to assess the efficacy of
AgNPs and $\mathrm{AgNO}_{3}$ against C. albicans-E. coli biofilms. Crystal violet provides a good measure of biofilm mass; it stains both bacteria cells and the extracellular matrix [22]. The crystal violet assay indicated that AgNPs was significantly effective in eliminating C. albicans-E. coli biofilms. The assay demonstrated the inhibition percentages of C. albicans-E. coli biofilms were $22.15 \%$ 
and $67.49 \%$ following the addition of 50 and $100 \mu \mathrm{L}$ of AgNPs, and $27.98 \%$ and $39.63 \%$ following the addition of 50 and $100 \mu \mathrm{L}$ of $\mathrm{AgNO}_{3}$, respectively. AgNPs and $\mathrm{AgNO}_{3}$ decreased C. albicans-E. coli biofilms compared with controls. $\mathrm{AgNO}_{3}$ and AgNPs were able to inhibit extracellular matrix growth, as shown in Fig. 2.

Furthermore, the XTT assay revealed that both $\mathrm{AgNO}_{3}$ and AgNPs reduced the number of constituent bacterial and fungal cells (Fig. 3). The XTT assay is the most commonly used assay to estimate viable cell count in biofilms and examine the impact of biofilm therapies [21]. As shown in Fig. 3, AgNPs and $\mathrm{AgNO}_{3}$ could decrease cell viability compared with controls. Cell viability was reduced as the concentrations of AgNPs and $\mathrm{AgNO}_{3}$ increased, with AgNPs being the most effective. Our data revealed that the inhibition percentages of cell viability were $53.14 \%$ and $96.53 \%$ following treatment with 50 and $100 \mu \mathrm{L}$ of AgNPs, and $51.46 \%$ and $60.51 \%$ following treatment with 50 and $100 \mu \mathrm{L}$ of $\mathrm{AgNO}_{3}$, respectively. These results were also supported by the CLSM assay data.
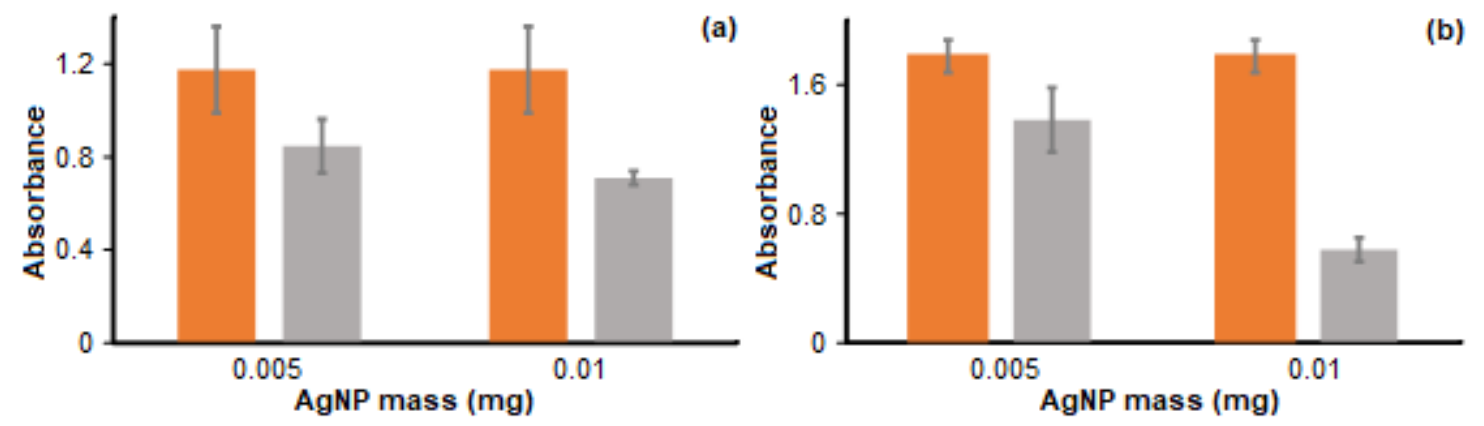

The CLSM assay demonstrated that the viable cell count of C. albicans and E. coli was reduced by penetrating $\mathrm{AgNO}_{3}$ and AgNPs in the extracellular matrix (Fig. 5 and 6). These assays indicated that the higher inhibition ability was proportional to the higher concentration of silver salts used. Thus, both $\mathrm{AgNO}_{3}$ and AgNPs have the potential to inhibit polymicrobial biofilms.

A noteworthy part of this research was the fact that AgNPs demonstrated more efficient antibiofilm activity compared with $\mathrm{AgNO}_{3}$. Also, the inhibition percentage of C. albicans-E. coli biofilms following treatment with $100 \mu \mathrm{L}$ of AgNPs was $67.49 \%$ compared with $96.53 \%$ on $\mathrm{AgNO}_{3}$ treatment. Furthermore, at a lower concentration, AgNPs showed better antibiofilm activity than $\mathrm{AgNO}_{3}$. This may be caused by several factors, including the size of AgNPs compare to that of $\mathrm{Ag}^{+}$.

Silver ions form a variety of size and motif clusters [23], which causes the formation of big-size structures. On the other hand, AgNPs comprise nano-sized structures formed of silver atoms that are metallically

Fig 2. The crystal violet assay of (a) AgNps and (b) $\mathrm{AgNO}_{3}$ against $C$. albicans-E. coli biofilm. The orange bar indicates the control group, and the grey bar indicates the treatment group. The measurement was performed in triplicates
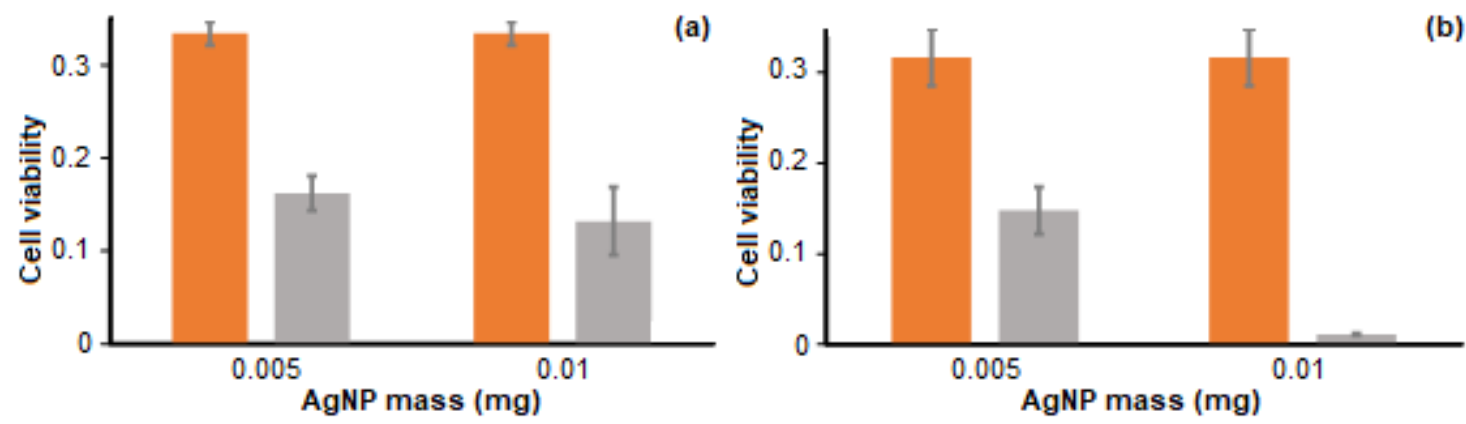

(b)

Fig 3. XTT assay of AgNPs (a) and $\mathrm{AgNO}_{3}$ (b) against C. albicans-E. coli biofilm. The orange bar indicates the control group, and the grey bar indicates the treatment group. The measurement was performed in triplicates 
bonded together and restricted in diameter from 1 to $100 \mathrm{~nm}$ by using a matrix. The silver ion will release from the matrix and easily entry to the extracellular matrix of biofilm to access microbial cells inside.

Microbes in a biofilm stay protected from external environmental threats owing to the presence of an extracellular matrix composed of polysaccharides and proteins. Thus, smaller antibiofilm molecules are needed to eliminate biofilms to penetrate the extracellular matrix. A previous study has reported that AgNPs are less toxic to humans compared with $\mathrm{AgNO}_{3}$. At low concentrations that are non-toxic to humans, the antibacterial ability of AgNPs is highlighted. Meanwhile, the $\mathrm{AgNO}_{3}$ concentration required to act as an antibacterial agent is toxic to humans [24].

Based on our previous description, we found that AgNPs were able to reduce the cell viability of $C$. albicansE. coli biofilms to a greater extent than $\mathrm{AgNO}_{3}$. Thus, we further analyzed AgNPs data using SEM. This assay was used to observe biofilm morphology with or without treatment with AgNPs. The typical morphology of $C$. albicans-E. coli biofilms was determined using the control group (Fig. 4(a)). C. albicans-E. coli biofilms that were grown on the cellulose nitrate membrane had aggregated cells covered by the extracellular matrix. Although both C. albicans and E. coli displayed a normal cell morphology, C. albicans was strongly associated with and aggregated to E. coli and was covered by the extracellular matrix. In accordance with previous research [25], SEM analysis in the present study verified that AgNPs are effective in degrading C. albicans-E. coli biofilms. Moreover, the treated C. albicans-E. coli biofilms demonstrated a decrease in the extracellular matrix compared to the untreated ones (Fig. 4(b)).

The degraded matrix and debris cells had holes that were formed as the result of matrix degradation. It has been reported that AgNPs can prevent extracellular matrix synthesis. This may explain the inability of certain microorganisms to form biofilms [25] (Scheme 1). In addition, no C. albicans and E. coli cells were found in C. albicans-E. coli biofilms treated with AgNPs (see Fig. 3(b)). This result followed that of the XTT assay, demonstrating the ability of AgNPs to reduce cell viability by approximately $96.53 \%$. This was because AgNPs possess the antimicrobial ability, which is mediated by the attachment of AgNPs to the microorganism's cell wall, penetrating it and killing the cells by cell membrane disruption [26]. SEM profile (Fig. 4) and crystal violet assay data (Fig. 2(a)) revealed the ability of AgNPs as extra cell-matrix removal. As for XTT assay, it has revealed the ability of AgNPs to act as antimicrobial toward both bacteria (E. coli) and fungi ( $C$. albicans). We conclude the dual function of AgNPs as an excellent extracellular matrix removal and cell killer in polymicrobial biofilms.

CLSM analysis was used to compare the differences among fluorescence intensities of C. albicans, E. coli, and the extracellular matrix from the AgNPs treatment and
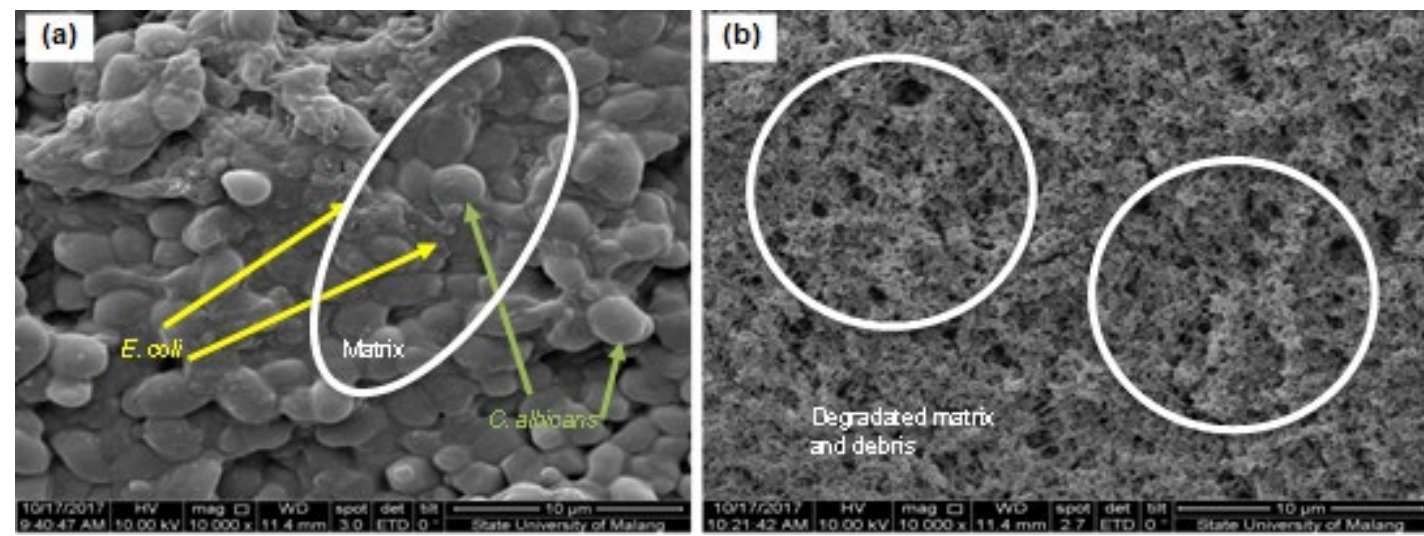

Fig 4. Scanning electron microscopy images of C. albicans-E. coli biofilm. (a) Control and (b) AgNP treatment. The surface of the extracellular matrix is circled in white. The degraded matrix and debris cells are circled in orange. The green arrows indicate C. albicans. The yellow arrows indicate hyphae 
control groups. Using CLSM assay, we observed the direct impact of AgNPs on C. albicans-E. coli biofilm components, including C. albicans, E. coli, and the extracellular matrix. This assay allowed us to reconstruct a three-dimensional structure of the biofilm by allowing the observation of all layers at different depths with spatial resolution in the order of micrometers [25]. This allowed the visualization of the state of each C. albicans-E. coli biofilm component using various dyes.

The AgNPs treatment group showed a decrease in fluorescence intensity, which can be observed as a weak fluorescence on the confocal image of the treatment group (Fig. 5 and 6) and in the quantitative data of mean intensity, which was decreased in the treatment group (Fig. $7(a)$ and $7(b)$ ). This reduced intensity was proportional to decreased cell numbers, as fluorescence was a consequence of specific binding between the dye and target. We used double staining with the SYTO-59 dye to visualize E. coli, with FITC to visualize C. albicans, and with Con A to visualize the extracellular matrix. Con A binds to mannose residues, producing a green color indicating the extracellular matrix [25]. Cell numbers are

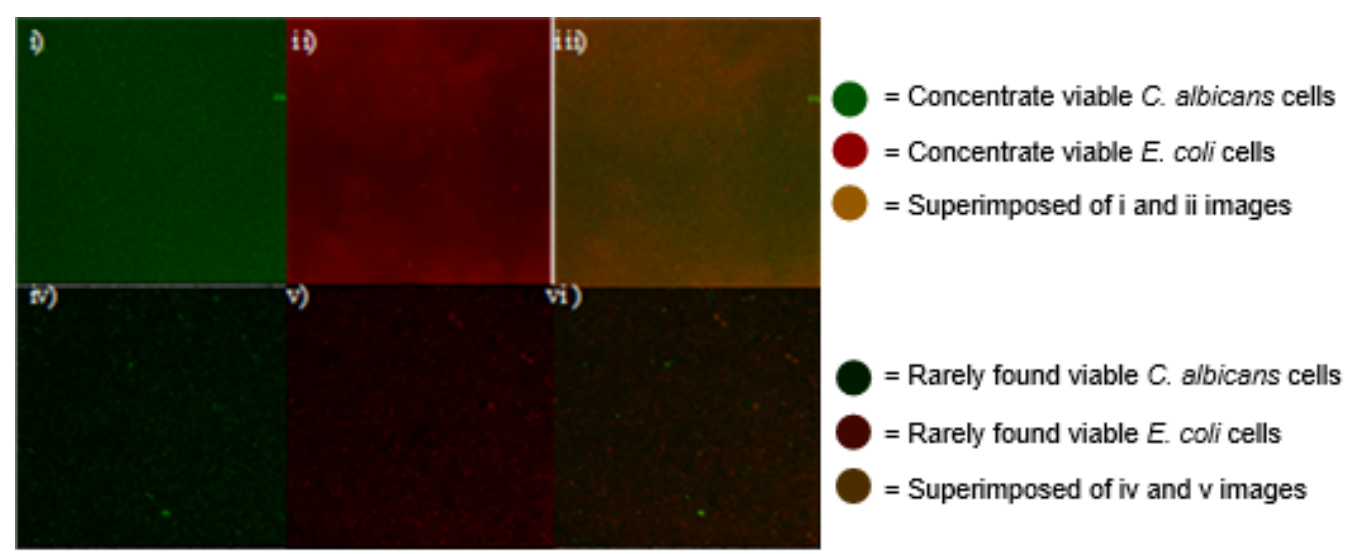

Fig 5. Confocal image of C. albicans-E. coli biofilms treated with AgNPs. SYTO-59 dye, FITC and Con A were used to visualize E. coli, C. albicans extracellular matrix, respectively. Control biofilm without treatment (i-iii) showed concentrate light dots of viable cells; As for AgNPs treated biofilm (iv-vi) showed only several light dots of viable cells; Therefore three images at the top (control biofilms without treatment) were far more luminescent than three images at the bottom AgNPs treated biofilms

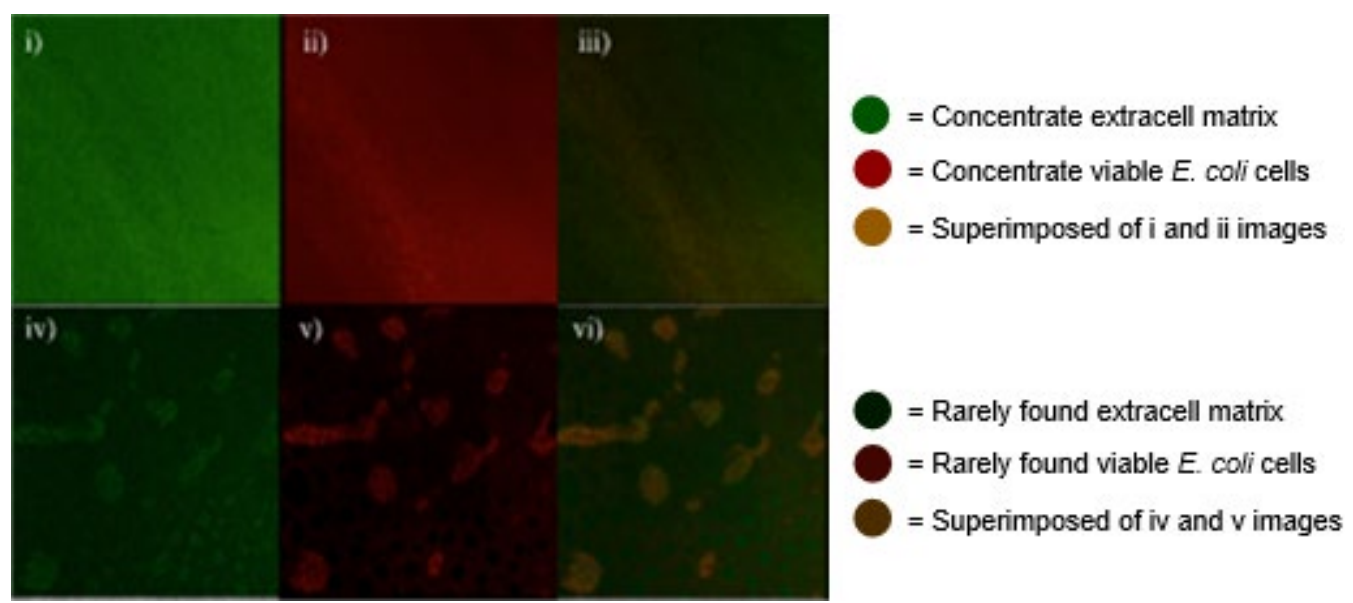

Fig 6. Confocal image of extra cell matrix and E. coli cells of C. albicans-E. coli biofilms treated with AgNPs. SYTO-59 dye to visualize E. coli and Con A to visualize the extracellular matrix. Control group without treatment (i-iii) showed concentrate light dots of extra cell matrix; As for treated biofilm (iv-vi) showed only several light dots of extra cell matrix and viable E. coli cells 
reflected by the intensity of fluorescence observed. We also observed the effect of treating E. coli cells and the extracellular matrix in C. albicans-E. coli biofilms using confocal imaging. We noted a decrease in $E$. coli cells and the extracellular matrix in the treatment group, as demonstrated by reduced staining intensities on the confocal image, compared with those in the control group (Fig. 6).

The existing water channels can explain the presence of dark areas within the biofilm, the heterogeneous production of the extracellular matrix, and the types of exopolysaccharides within the biofilm, and the absence of Con A-FITC binding to the matrix [25]. When CLSM images with red and green fluorescent intensities were superimposed, yellow-colored areas (green + red) revealed that the extracellular-Con A-FITC-reactive polysaccharide (green) was produced in intracellular spaces (red), indicating that extracellular polysaccharides were formed as a capsular component in these biofilms [25].

In addition, we identified that in the AgNPs treatment group, the cell numbers of $C$. albicans and $E$. coli reduced (Fig. 5), as assessed using reduced fluorescence intensities, compared with that in the control group (Fig. 4(a)); this finding was further supported by quantitative data (Fig. 7), showed the cell numbers of $C$. albicans and E. coli in the AgNPs treatment group decreased by 57.93 and $50.54 \%$, respectively, compared with that in the control group. In the control group, the cell numbers of C. albicans were less than those of E. coli. This is possibly due to the difference in the growth abilities of $C$. albicans and E. coli within the same period.

Fig. 6 shows E. coli and the extracellular matrix in
C. albicans-E. coli biofilm. The decreased cell number and extracellular matrix (Fig. 7(b)) observed in the AgNPs treatment group were due to reduced fluorescence intensity compared with that in the control group. This result was also following our quantitative data. The extracellular matrix and the cell number of $E$. coli decreased by 41.89 and $56.81 \%$, respectively. Thus, we conclude that AgNPs can reduce both the number of bacterial cells and the extracellular matrix. The extracellular matrix acts as a protective structure in the biofilm, making it resistant to treatment. The extracellular matrix remains a factor contributing to biofilm resistance against antibiotics and other drugs. According to our quantitative data, AgNPs may be a potential alternative solution to eliminate the extracellular matrix and act as an antimicrobial agent. This dual function of AgNPs confers it with excellent antibiofilm abilities. AgNPs may act via attachment to biofilms, thus inhibiting their growth.

Furthermore, AgNPs can pass through the biofilm water channel. Biofilm water channels play a role in transferring nutrition from the outer environment to the cells beneath the extracellular matrix. AgNPs may pass through these channels due to their small particle size, thus allowing microbial cell access to implement antimicrobial effect and inhibit biofilm formation [2526]. In addition, AgNPs have a high affinity to microbial cell membranes, thus penetrating and accumulating inside cells and continuously releasing silver ions and damaging the cells [27]. These features of AgNPs contribute to their roles in degrading the extracellular matrix and eliminating E. coli and C. albicans.
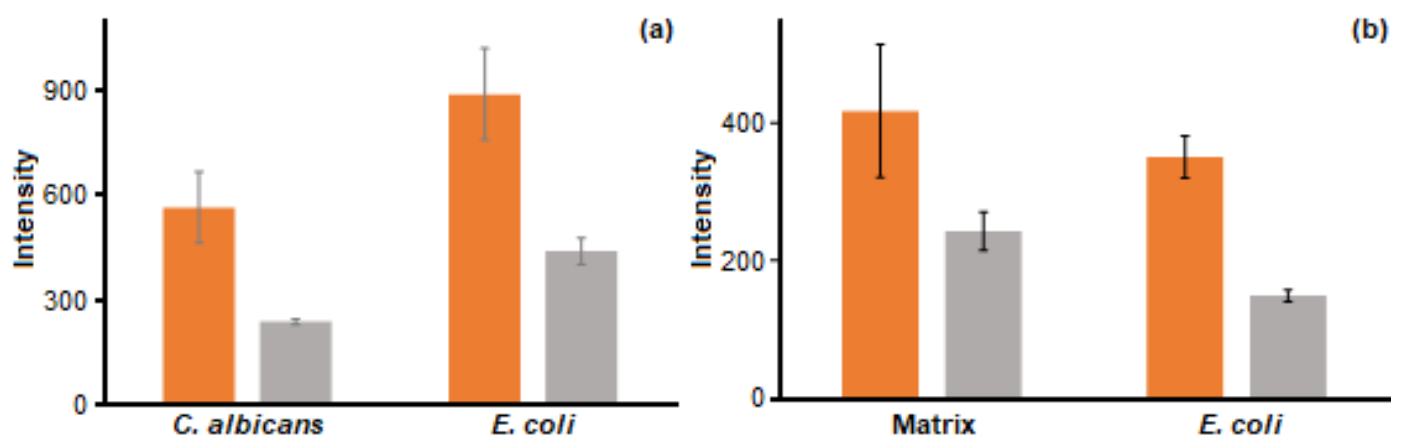

Fig 7. Quantitative data obtained using the CLSM of C. albicans-E. coli biofilm. The orange bar indicates the control group, and the grey bar indicates the treatment group. The measurements were performed in triplicates 


\section{- CONCLUSION}

AgNPs can reduce the number of bacterial cells (acts as cells killer) and degrade the extracellular matrix (extracellular matrix removal). As a cell killer, the percent inhibition cell viability of $C$. albicans-E. coli inside matrix biofilms following treatment with $100 \mu \mathrm{L}$ of AgNPs was 96.53\%. As matrix extra cell removal, AgNPs remove $56.81 \%$ of the extracellular matrix covering the cells. These findings suggest that AgNPs can be developed to become potent anti-biofilms towards polymicrobial biofilm consist of $C$. albicans and other pathogenic bacteria. AgNPs can omit tight and thick extracellular matrices that blocking antibiotics reaches their target in the cell, which is one of the triggers of multidrug resistance.

\section{- ACKNOWLEDGMENTS}

The authors thank the Ministry of Research, Technology, and Higher Education for financial support on this study and Airlangga University for the meaningful supports.

\section{- REFERENCES}

[1] Rabin, N., Zheng, Y., Opoku-Temeng, C., Du, Y., Bonsu, E., and Sintim, H.O., 2015, Biofilm formation mechanisms and targets for developing antibiofilm agents, Future Med. Chem., 7 (4), 493-512.

[2] Gulati, M., and Nobile, C.J., 2016, Candida albicans biofilms: Development, regulation, and molecular mechanisms, Microbes Infect., 18 (5), 310-321.

[3] Burmølle, M., Ren, D., Bjarnsholt, T., and Sørensen, S.J., 2014, Interactions in multispecies biofilms: Do they actually matter?, Trends Microbiol., 22 (2), 84-91.

[4] Nobile, C.J., Johnson, A.D., 2015, Candida albicans biofilms and human disease, Annu. Rev. Microbiol., 69, 71-92.

[5] Bandara, H.M.H.N., Yau, J.Y.Y., Watt, R.M., Jin, L.J., and Samaranayake, L.P., 2009, Escherichia coli and its lipopolysaccharide modulate in vitro Candida biofilm formation, J. Med. Microbiol., 58 (12), 1623-1631.

[6] Palanisamy, N.K., Ferina, N., Amirulhusni, A.N., Mohd-Zain, Z., Hussaini, J., Ping, L.J., and Durairaj, R., 2014, Antibiofilm properties of chemically synthesized silver nanoparticles found against Pseudomonas aeruginosa, J. Nanobiotechnol., 12 (1), 2.

[7] Monroe, D., 2007, Looking for chinks in the armor of bacterial biofilms, PLoS Biol., 5 (11), e307.

[8] Peters, B.M., Jabra-Rizk, M.A., O’May, G.A., Costerton, J.W., and Shirtliff, M.E., 2012, Polymicrobial interactions: Impact on pathogenesis and human disease, Clin. Microbiol. Rev., 25 (1), 193-213.

[9] dos Santos, J.D., Piva, E., Vilela, S.F.G., Jorge, A.O.C., and Junqueira, J.C., 2016, Mixed biofilms formed by C. albicans and non-albicans species: A study of microbial interactions, Braz. Oral Res., 30, e23.

[10] Baktir, A., Masfufatun, Hanum, G.R., Amailia, K.R., and Purkan, 2014, Construction and characterization of the intestinal biofilm model of Candida spp, Res. J. Pharm., Biol. Chem. Sci., 5 (1), 204-211.

[11] Harriott, M.M., and Noverr, M.C., 2011, Importance of Candida-bacterial polymicrobial biofilms in disease, Trends Microbiol., 19 (11), 557-563.

[12] Røder, H.L., Sørensen, S.J., and Burmølle, M., 2016, Studying bacterial multispecies biofilms: Where to start?, Trends Microbiol., 24 (6), 503-513.

[13] Fox, E.P., and Nobile, C.J., 2012, A sticky situation: Untangling the transcriptional network controlling biofilm development in Candida albicans, Transcription, 3 (6), 315-22.

[14] Vandecandelaere, I., Matthijs, N., Nelis, H.J., Depuydt, P., and Coenye, T., 2013, The presence of antibiotic-resistant nosocomial pathogens in endotracheal tube biofilms and corresponding surveillance cultures, Pathog. Dis., 69 (2), 142-148.

[15] Samaranayake, Y.H., Bandara, H.M.H.N., Cheung, B.P.K., Yau, J.Y.Y., Yeung, S.K.W., and Samaranayake, L.P., 2014, Enteric gram-negative bacilli suppress Candida biofilms on Foley urinary catheters, APMIS, 122 (1), 47-58.

[16] De Brucker, K., Tan, Y., Vints, K., De Cremer, K., Braem, A., Verstraeten, N., Michiels, J., Vleugels, J., Cammue, B.P.A., and Thevissen, K., 2015, Fungal 
$\beta$-1,3-glucan increases ofloxacin tolerance of Escherichia coli in a polymicrobial E. coli/Candida albicans biofilm, Antimicrob. Agents Chemother., 56 (9), 3052-3058.

[17] Ansari, M.A., Khan, H.M., Khan, A.A., Cameotra, S.S., and Pal, R., 2013, Antibiofilm efficacy of silver nanoparticles against biofilm of extended spectrum $\beta$-lactamase isolates of Escherichia coli and Klebsiella pneumoniae, Appl. Nanosci., 4 (7), 859-868.

[18] Panáček, A., Kolář, M., Večeřová, R., Prucek, R., Soukupová, J., Kryštof, V., Hamal, P., Zbořil, R., and Kvítek, L., 2009, Antifungal activity of silver nanoparticles against Candida spp., Biomaterials, 30 (31), 6333-6340.

[19] Li, W.R., Xie, X.B., Shi, Q.S., Zeng, H.Y., Ou-Yang, Y.S., and Chen, Y.B., 2010, Antibacterial activity and mechanism of silver nanoparticles on Escherichia coli, Appl. Microbiol. Biotechnol., 85 (4), 1115-1122.

[20] Abishek, B., and Hemlata, C., 2014, Antibacterial activity of silver nanoparticles conjugated with antibiotics, Bionano Front., 7, 32-35.

[21] McGillicuddy, E., Murray, I., Kavanagh, S., Morrison, L., Fogarty, A., Cormican, M., Dockery, P., Prendergast, M., Rowan, N., and Morris, D., 2017, Silver nanoparticles in the environment: Sources, detection and ecotoxicology, Sci. Total Environ., 575, 231-246.
[22] Manikprabhu, D., and Lingappa, K., 2013, Microwave assisted rapid and green synthesis of silver nanoparticles using a pigment produced by Streptomyces coelicolor klmp33, Bioinorg. Chem. Appl., 2013, 341798.

[23] Blom, M.N., Schooss, D., Stairs, J., and Kappes, M.M., 2006, Experimental structure determination of silver cluster ions $\left(\mathrm{Ag}_{\mathrm{n}}{ }^{+}, 19 \leq \mathrm{n} \leq 79\right)$, J. Chem. Phys., 124 (24), 244308.

[24] Kvitek, L., Panacek, A., Prucek, R., Soukupova, J., Vanickova, M., Kolar, M., and Zboril, R., 2011, Antibacterial activity and toxicity of silver nanosilver versus ionic silver, J. Phys. Conf. Ser., 304, 012029.

[25] Welch, K., Cai, Y., and Strømme, M., 2012, A method for quantitative determination of biofilm viability, J. Funct. Biomater., 3 (2), 418-431.

[26] Nett, J.E., Cain, M.T., Crawford, K., and Andes, D.R., 2011, Optimizing a Candida biofilm microtiter plate model for measurement of antifungal susceptibility by tetrazolium salt assay, $J$. Clin. Microbiol., 49, 1426-33.

[27] Ansari, M.A., Khan, H.M., Khan, A.A., Cameotra, S.S., and Alzohairy, M.A., 2015, Anti-biofilm efficacy of silver nanoparticles against MRSA and MRSE isolated from wounds in a tertiary care hospital, Indian J. Med. Microbiol., 33 (1), 101-109. 